\title{
Elastodynamic Impact into Piezoelectric Media
}

by George A Gazonas, Raymond A Wildman, and David A Hopkins

ARL-TR-7056

September 2014 


\section{NOTICES}

\section{Disclaimers}

The findings in this report are not to be construed as an official Department of the Army position unless so designated by other authorized documents.

Citation of manufacturer's or trade names does not constitute an official endorsement or approval of the use thereof.

Destroy this report when it is no longer needed. Do not return it to the originator. 


\title{
Army Research Laboratory
}

Aberdeen Proving Ground, MD 21005-5066

\section{Elastodynamic Impact into Piezoelectric Media}

\author{
George A Gazonas, Raymond A Wildman, and David A Hopkins
}

Weapons and Materials Research Directorate, ARL 


\section{REPORT DOCUMENTATION PAGE}

Public reporting burden for this collection of information is estimated to average 1 hour per response, including the time for reviewing instructions, searching existing data sources, gathering and maintaining the data needed, and completing and reviewing the collection information. Send comments regarding this burden estimate or any other aspect of this collection of information, including suggestions for reducing the burden, to Department of Defense, Washington Headquarters Services, Directorate for Information Operations and Reports (0704-0188), 1215 Jefferson Davis Highway, Suite 1204, Arlington, VA 22202-4302. Respondents should be aware that notwithstanding any other provision of law, no person shall be subject to any penalty for failing to comply with a collection of information if it does not display a currently valid OMB control number.

PLEASE DO NOT RETURN YOUR FORM TO THE ABOVE ADDRESS.

\begin{tabular}{|l|l|l|}
\hline 1. REPORT DATE (DD-MM- $Y Y Y Y)$ & 2. REPORT TYPE & 3. DATES COVERED (From - To)
\end{tabular}

\begin{tabular}{l|l} 
September 2014 & Final \\
\hline
\end{tabular}

4. TITLE AND SUBTITLE

Elastodynamic Impact into Piezoelectric Media July 2013-July 2014

5a. CONTRACT NUMBER

5b. GRANT NUMBER

5c. PROGRAM ELEMENT NUMBER

6. AUTHOR(S)

George A Gazonas, Raymond A Wildman, and David A Hopkins

5d. PROJECT NUMBER

AH84

5e. TASK NUMBER

5f. WORK UNIT NUMBER

7. PERFORMING ORGANIZATION NAME(S) AND ADDRESS(ES)

U.S. Army Research Laboratory

ATTN: RDRL-WMM-B

Aberdeen Proving Ground, MD 21005-5069

9. SPONSORING/MONITORING AGENCY NAME(S) AND ADDRESS(ES)

8. PERFORMING ORGANIZATION REPORT NUMBER

ARL-TR-7056

10. SPONSOR/MONITOR'S ACRONYM(S)

11. SPONSOR/MONITOR'S REPORT NUMBER(S)

\section{DISTRIBUTION/AVAILABILITY STATEMENT}

Approved for public release; distribution is unlimited.

13. SUPPLEMENTARY NOTES

primary author's email: <george.a.gazonas.civ@mail.mil>

\section{ABSTRACT}

We consider the elastodynamic impact problem involving a one-dimensional (1-D) semi-infinite non-piezoelectric elastic flyer traveling at initial velocity $V_{0}$ that collides and welds to a finite stationary piezoelectric target backed by a semi-infinite non-piezoelectric elastic half-space. A combined d'Alembert and Laplace transform method is used to derive new numerically based solutions for this class of transient wave propagation problems. A modified Dubner-Abate-Crump (DAC) algorithm is used invert the analytical Laplace domain solutions to the time domain. Unlike many authors who neglect electromechanical coupling in the initially unstressed region ahead of the shock, we consider this effect, which gives rise to the development of a tensile stress wave within the piezoelectric target ahead of the shock. The final value theorem (FVT) is used to derive an explicit expression for the asymptotic (long-time) displacement current in the target. We also apply a newly derived impact boundary condition to the problem of elastodynamic impact into a lead zirconate titanate (PZT-4) target and find that the solutions obtained using the modified-DAC algorithm compare well with those obtained using both finite-difference time-domain (FDTD) methods, and the commercial finite element code, COMSOL Multiphysics.

15. SUBJECT TERMS Numerical inverse Laplace transform; modified Dubner-Abate-Crump algorithm; impact boundary condition; FDTD; COMSOL Multiphysics; Mathematica; verification of numerical codes; wave propagation

\begin{tabular}{|c|c|c|c|c|c|}
\hline \multicolumn{3}{|c|}{ 16. SECURITY CLASSIFICATION OF: } & $\begin{array}{l}\text { 17. LIMITATION } \\
\text { OF ABSTRACT }\end{array}$ & $\begin{array}{l}\text { 18. NUMBER } \\
\text { OF PAGES }\end{array}$ & $\begin{array}{l}\text { 19a. NAME OF RESPONSIBLE PERSON } \\
\text { George A. Gazonas }\end{array}$ \\
\hline $\begin{array}{l}\text { a. REPORT } \\
\text { Unclassified }\end{array}$ & $\begin{array}{l}\text { b. ABSTRACT } \\
\text { Unclassified }\end{array}$ & $\begin{array}{l}\text { c. THIS PAGE } \\
\text { Unclassified }\end{array}$ & UU & 30 & $\begin{array}{l}\text { 19b. TELEPHONE NUMBER (Include area code) } \\
\text { 410-306-0863 }\end{array}$ \\
\hline
\end{tabular}




\section{Contents}

List of Figures

List of Tables $\quad$ v

$\begin{array}{ll}\text { 1. Introduction } & 1\end{array}$

2. Plane Waves in Piezoelectric Media 2

3. Impact-Induced Plane Waves in a Piezoelectric Target 3

4. Laplace Transform of the D'Alembert Solution $\quad 5$

5. Discussion and Conclusions 11

$\begin{array}{lr}\text { 6. References } & 12\end{array}$

Appendix. Mathematica Code for Determining the Numerical ILT Using the Modified$\begin{array}{ll}\text { DAC Algorithm } & 15\end{array}$

$\begin{array}{ll}\text { Distribution List } & 18\end{array}$ 


\section{List of Figures}

Fig. 1 A semi-infinite non-piezoelectric elastic flyer (impedance $z_{0}$ ) traveling at initial velocity $V_{0}$ impacting a piezoelectric target (impedance $z_{1}$ ) of length $l$; the short-circuited target $V_{1}(t)=0$ is backed by a semi-infinite non-piezoelectric elastic

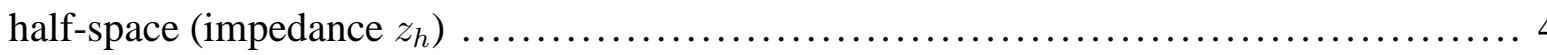

Fig. 2 Normalized stress history at the center of a 5- $m m$-thick piezoelectric target (PZT-4) backed by a semi-infinite non-piezoelectric elastic half-space under impact by a semi-infinite non-piezoelectric elastic flyer with initial velocity $V_{0}=5 \mathrm{~m} / \mathrm{s}$; the normalization factor is the initial elastic stress jump at impact given by $\sigma\left(0,0^{+}\right)=\frac{z_{0} z_{1 E}}{z_{0}+z_{1 E}} V_{0}$ where $z_{1 E}$ is the "elastic-only" impedance of the target, and $z_{1 E}=\rho_{1} c_{1 E}=7500 \times 3922.8 c_{1 E}=\sqrt{C^{E} / \rho_{1}}$, see Table 1 8

Fig. 3 Normalized velocity history at the center of a 5- $m m$-thick piezoelectric target (PZT-4) backed by a semi-infinite non-piezoelectric elastic half-space under impact by a semi-infinite non-piezoelectric elastic flyer with initial velocity $V_{0}=5 \mathrm{~m} / \mathrm{s}$; the normalization factor is the initial elastic velocity jump at impact given by $v\left(0,0^{+}\right)=\frac{z_{0}}{z_{0}+z_{1 E}} V_{0}$, where $z_{1 E}$ is the "elastic-only" impedance of the target, and $z_{1 E}=\rho_{1} c_{1 E}=7500 \times 3922.8 c_{1 E}=\sqrt{C^{E} / \rho_{1}}$, see Table 1

Fig. 4 Displacement current time history at the center of a 5-mm-thick piezoelectric target (PZT-4) backed by a semi-infinite non-piezoelectric elastic half-space under impact by a semi-infinite non-piezoelectric elastic flyer with initial velocity $V_{0}=5 \mathrm{~m} / \mathrm{s}$; the asymptotic displacement current $D_{1}(\infty)=-\frac{z_{0} z_{h} h \epsilon}{\left(z_{0}+z_{h}\right)\left(c_{1} z_{1}-h^{2} \epsilon\right)} V_{0}=-0.0131$

Fig. A-1 Normalized stress time history at the center of a 5- $\mathrm{mm}$-thick piezoelectric target (PZT-4) backed by a semi-infinite non-piezoelectric elastic half-space under impact by a semi-infinite non-piezoelectric elastic flyer with initial velocity $V_{0}=5 \mathrm{~m} / \mathrm{s}$ 


\section{List of Tables}

Table $1 \quad$ Mechanical and electrical properties ................................. 7 
INTENTIONALLY LEFT BLANK. 


\section{Introduction}

Much of the literature that presents analytical or computational solutions to "impact-type" initial boundary value problems assumes an impact boundary condition in the form of a constant applied step in stress or velocity, $\mathrm{cf}^{1-3}$ This simplifying assumption is particularly useful for characterizing elastic shocks in flyers and targets that are homogeneous, linear elastic, and semi-infinite. Here, elastic shocks are defined by stress wave amplitudes that are less than or equal to the Hugoniot elastic limit, cf., page 248, equation (10.4) of Davison. ${ }^{4}$ However, for a true impact into a piezoelectric medium that is multilayered, or inhomogeneous, or possesses other material discontinuities, the target introduces multiple reflected or scattered waves at the impact face, so that the history of the stress $\sigma$ and the particle velocity $v$ at the impact face is generally time-dependent and cannot be known in advance of the solution to the problem. The use of a constant stress or velocity boundary condition is an invalid assumption for modeling impact in such targets, yet its use is widespread in the mechanics literature.

Both Redwood ${ }^{5}$ and Steutzer ${ }^{6}$ study the problem of multiply reflected transient waves, characterized by an infinite set of jump discontinuities, in linear piezoelectrics subjected to either a step in stress or voltage, but these boundary conditions are not appropriate for the elastodynamic impact problem. Other works address shock wave propagation in nonlinear piezoelectric media, ${ }^{7-9}$ but the complexity of the material response prohibits an analytical treatment of the electromechanical coupling in the unstressed region ahead of the shock wave. In this report, we first outline the linear equations of dynamic piezoelectricity, following closely the development found in Redwood ${ }^{5}$ and Steutzer, ${ }^{6}$ and show how the d'Alembert solution is also applicable to the study of multiply reflected transient waves in linear piezoelectrics. We then study the "true" elastodynamic impact problem using a new impact boundary condition originally introduced by Scheidler and Gazonas; ${ }^{10}$ the full derivation of the impact boundary condition is based on the d'Alembert solution of the wave equation, and appears in Gazonas et al. ${ }^{11}$ Our results are analytical in the Laplace domain but are numerically inverted to the time domain using a modified Dubner-Abate-Crump (DAC) algorithm ${ }^{12}$ designed to mitigate the effects (using Lanczos $\sigma$-factors) of the Gibbs phenomenon (ringing seen in Fourier series representations of jump discontinuities). One-dimensional (1-D) impact-induced stress, velocity, and electrical displacement fields at the center of a lead zirconate titanate (PZT-4) target are compared with solutions obtained using a finite-difference time-domain (FDTD) code and the commercial finite element (FE) code, COMSOL Multiphysics. ${ }^{13}$ The FDTD code was developed by Raymond A 
Wildman and the COMSOL simulations were performed by David A Hopkins to compare with the derived numerical inverse Laplace transform (ILT) solutions. The final value theorem (FVT) can be used to verify that the asymptotic (long-time) stress and velocity in the piezoelectric target are independent of the target's elastic or piezoelectric properties, and only the elastic properties of the flyer and half-space, a result also proven using the time-domain solutions obtained for purely elastic targets. ${ }^{11}$ The FVT is also used to determine a closed-form expression for the asymptotic displacement current.

\section{Plane Waves in Piezoelectric Media}

The 1-D constitutive equations for a linear piezoelectric material can be written using the notation $^{5,14}$

$$
\begin{gathered}
\sigma(x, t)=C^{D} \frac{\partial u(x, t)}{\partial x}-h D(x, t), \\
\mathcal{E}(x, t)=-h \frac{\partial u(x, t)}{\partial x}+D(x, t) / \epsilon,
\end{gathered}
$$

where $\sigma$ is the stress in $N / m^{2}, C^{D}$ is Young's modulus measured at constant $D$ in $N / m^{2}, u$ is the particle displacement in $m, D$ is the electric flux density in $C / m^{2}, \epsilon=\epsilon_{0} \epsilon_{r}$ is the dielectric permittivity in $F / m, \epsilon_{0}$ is the permittivity of a vacuum in $F / m, \epsilon_{r}$ is the relative permittivity of the medium, $h$ is a piezoelectric constant measured at constant $D$ in $V / m$, and $\mathcal{E}$ is the electric field in $V / m$ (or $N / C$ ). The piezoelectrically stiffened elastic constant $C^{D}$, Eq. 1 , is defined in Cady $^{15}$ (pg. 270 equation (271 a)), by The Institute of Radio Engineers (IRE) ${ }^{14}$ (pg. 1394, Table III) or even Chen et al. ${ }^{1}$ (pg. 4760), and is given by

$$
C^{D}=C^{E}+e h
$$

where $C^{E}$ is Young's modulus measured at constant $\mathcal{E}, e$ is a piezoelectric coupling constant in $C / m^{2}$, and $h=e / \epsilon$. In addition, from Newton's second law,

$$
\rho \frac{\partial^{2} u(x, t)}{\partial t^{2}}=\frac{\partial \sigma(x, t)}{\partial x}, \text { for } t>0, \quad x \in(-\infty, \infty)
$$

where $\rho$ is the mass density in $\mathrm{kg} / \mathrm{m}^{3}$. Following the development in $\operatorname{Redwood}^{5}$ or Steutzer ${ }^{6}$ for 
linear piezoelectric media, substitution of Eq. 1 into Eq. 4 gives

$$
\frac{\partial^{2} u(x, t)}{\partial t^{2}}=\frac{C^{D}}{\rho} \frac{\partial^{2} u(x, t)}{\partial x^{2}}-\frac{h}{\rho} \frac{\partial D(x, t)}{\partial x} .
$$

If there is no free charge, $\rho_{\text {free }}=0$, inside the piezoelectric medium, then $\operatorname{div} D=\rho_{\text {free }}=0$ (see e.g., Lorrain and Corson, ${ }^{16} \mathrm{pg}$. 109), and the differentials with respect to $y$ and $z$ are assumed to be zero (plane wave propagation in Cartesian coordinates), $d D_{y} / d x=d D_{z} / d x=0$, hence $d D_{x} / d x=0$, and Eq. 5 becomes

$$
\frac{\partial^{2} u(x, t)}{\partial t^{2}}=c^{2} \frac{\partial^{2} u(x, t)}{\partial x^{2}}
$$

where $c=\sqrt{C^{D} / \rho}$ is the piezo-modified longitudinal wave speed with $C^{D}$ given by Eq. 3 .

\section{Impact-Induced Plane Waves in a Piezoelectric Target}

We now consider the problem of a semi-infinite non-piezoelectric elastic flyer traveling at initial velocity $V_{0}$ that collides with (and welds to) a finite, undeformed, stationary piezoelectric target of finite length $l$; the target is backed by a semi-infinite non-piezoelectric elastic half-space (Fig. 1). Because our sign convention for stress is taken positive in compression, the stress $\sigma_{1}$ in the linear elastic piezoelectric target is given by Eq. 1 with the minus sign:

$$
\sigma_{1}(x, t)=-C_{1}^{D} \frac{\partial u_{1}(x, t)}{\partial x}+h D_{1}(x, t)
$$

We denote field quantities with a subscript of " 1 " as quantities in the piezoelectric target, and subscripts of " 0 " and " $h$ " refer to field variables in the flyer and half-space, respectively. From Eq. 6 with this notation, the transient target displacements $u_{1}(x, t)$ are governed by the wave equation in one-spatial dimension,

$$
\frac{\partial^{2} u_{1}(x, t)}{\partial t^{2}}=c_{1}^{2} \frac{\partial^{2} u_{1}(x, t)}{\partial x^{2}}, \text { for } t>0, \quad x \in(0, l)
$$

The general solution to the wave Eq. 8 in the target can be constructed using d'Alembert's equation:

$$
u_{1}(x, t)=f_{1}\left(t-x / c_{1}\right)+g_{1}\left(t+x / c_{1}\right)
$$

where $f_{1}(\cdot)$ and $g_{1}(\cdot)$ are arbitrary functions. The displacement $u_{1}$ is assumed to be continuous 


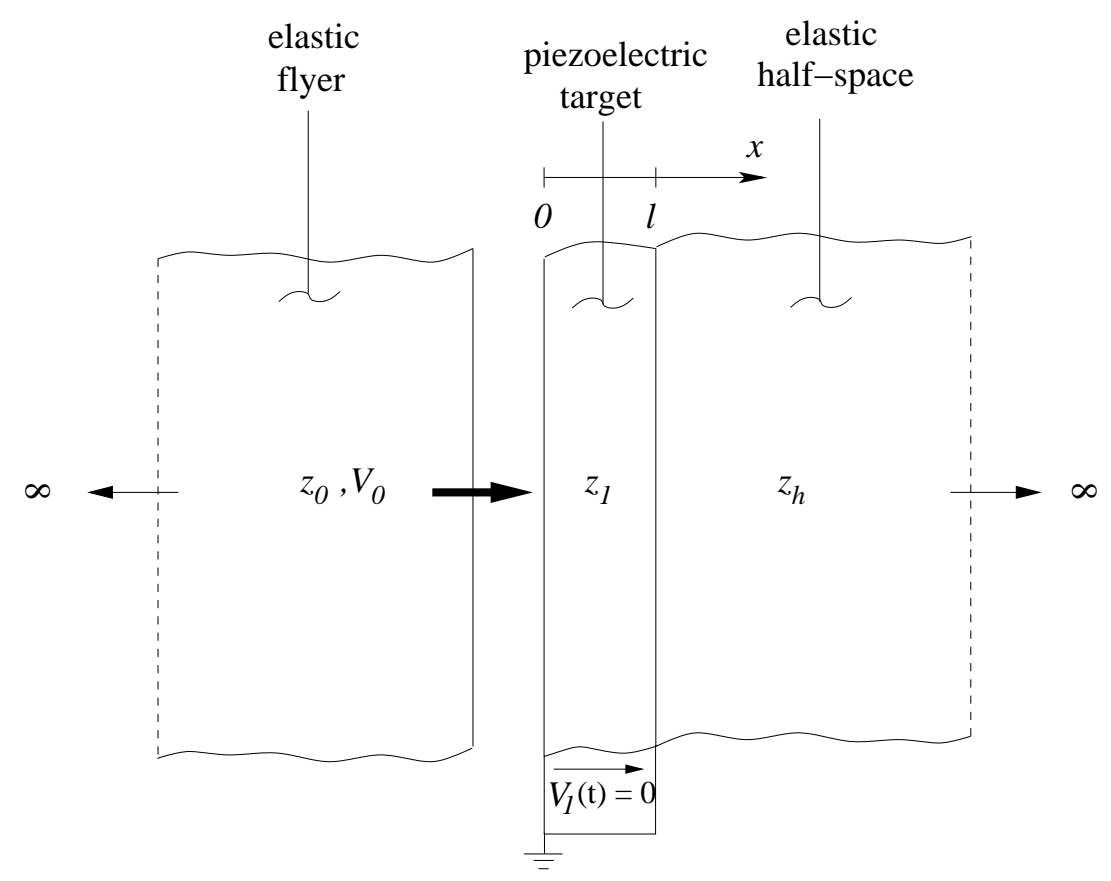

Fig. 1. A semi-infinite non-piezoelectric elastic flyer (impedance $z_{0}$ ) traveling at initial velocity $V_{0}$ impacting a piezoelectric target (impedance $z_{1}$ ) of length $l$; the short-circuited target $V_{1}(t)=0$ is backed by a semi-infinite non-piezoelectric elastic half-space (impedance $z_{h}$ ).

and piecewise-smooth, which implies that the d'Alembert functions $f_{1}$ and $g_{1}$ are also continuous and piecewise-smooth. Letting $f_{1}^{\prime}=F_{1}$ and $g_{1}^{\prime}=G_{1}$, then the d'Alembert solution 9, together with the Eq. 7 for the stress, yields

$$
\sigma_{1}(x, t)=z_{1}\left[F_{1}\left(t-x / c_{1}\right)-G_{1}\left(t+x / c_{1}\right)\right]+h D_{1}(x, t),
$$

where $z_{1}=\rho_{1} c_{1}$ is the piezo-modified target impedance. Similarly, the particle velocity $v_{1}$ in the target is given by

$$
v_{1}(x, t)=\frac{\partial u_{1}}{\partial t}=F_{1}\left(t-x / c_{1}\right)+G_{1}\left(t+x / c_{1}\right) .
$$

It is evident that the stress and particle velocity in the target are determined once the functions $F_{1}, G_{1}$, and $D_{1}$ are determined. Note that discontinuities in $F_{1}$ or $G_{1}$ correspond to the passage of shock waves at interior points or the reflection of shock waves at the boundaries $x=0$ and $x=l$. This behavior is independent of the back face boundary conditions, assuming the flyer welds to the target at impact. 
To solve for $F_{1}, G_{1}$, and $D_{1}$ requires consideration of the initial conditions (in this case homogeneous) and boundary conditions of the target. The initial conditions are

$$
u_{1}(x, 0)=0, \quad \frac{\partial u_{1}(x, 0)}{\partial t}=v_{1}(x, 0)=0, x \in[0, l]
$$

Then Eq. $12_{1}$ and the stress-strain Eq. 7 imply that

$$
\sigma_{1}(x, 0)=D_{1}(x, 0)=0, \quad x \in[0, l]
$$

\section{Laplace Transform of the D'Alembert Solution}

Solutions for the true impact problem with appropriate back face boundary conditions are obtained in this section by Laplace transform techniques. The derivation of these solutions require the Laplace transform of the field equations, which is considered here. Let $f$ denote a real-valued, piecewise-continuous function defined at least on $[0, \infty)$. We employ the right-sided or unilateral Laplace transform $\mathcal{L}$ of $f$ defined as

$$
\bar{f}(s)=\mathcal{L}\{f\}(s)=\mathcal{L}_{t}\{f(t)\}(s) \equiv \int_{0}^{\infty} f(t) e^{-s t} d t
$$

Here $s=\sigma+\mathrm{i} \omega$ is generally a complex number with $\Re(s)>0$, and the overbar on $f$ indicates that the function is in the Laplace domain. The Laplace transforms of field variables such as $u_{1}(x, t), \sigma_{1}(x, t)$ and $v_{1}(x, t)$ are taken with respect to the time variable $t$, for example,

$$
\bar{\sigma}_{1}(x, s)=\mathcal{L}_{t}\left\{\sigma_{1}(x, t)\right\}(s)=\int_{0}^{\infty} \sigma_{1}(x, t) e^{-s t} d t
$$

Applying Laplace's shifting theorem, we see that

$$
\mathcal{L}_{t}\left\{F_{1}\left(t+x / c_{1}\right)\right\}=e^{\frac{s x}{c_{1}}} \bar{F}_{1}(s), \quad \mathcal{L}_{t}\left\{G_{1}\left(t-x / c_{1}\right)\right\}=e^{\frac{-s x}{c_{1}}} \bar{G}_{1}(s)
$$

Laplace transformation of Eqs. 9, 10, and 11, and using Eq. 16, we obtain Laplace-domain expressions for the displacement, stress, and particle velocity in the target in terms of the 
transforms of the d'Alembert functions:

$$
\begin{aligned}
& \bar{u}_{1}(x, s)=\frac{1}{s}\left[e^{\frac{-s x}{c_{1}}} \bar{F}_{1}(s)+e^{\frac{s x}{c_{1}}} \bar{G}_{1}(s)\right] \\
& \bar{\sigma}_{1}(x, s)=z_{1}\left[e^{\frac{-s x}{c_{1}}} \bar{F}_{1}(s)-e^{\frac{s x}{c_{1}}} \bar{G}_{1}(s)\right]+h \bar{D}_{1}(s) \\
& \bar{v}_{1}(x, s)=\left[e^{\frac{-s x}{c_{1}}} \bar{F}_{1}(s)+e^{\frac{s x}{c_{1}}} \bar{G}_{1}(s)\right] .
\end{aligned}
$$

To find the potential across the piezoelectric target of thickness, $l$, integrate the electric field $\mathcal{E}_{1}=-\nabla V_{1}$. Unlike the gravitational field and other field potentials, the minus sign is used here since the electric field is "governed by Coulomb's law where elements of like sign repel," cf., page 22 of Sternberg and Smith. ${ }^{17}$

$$
V_{1}(t)=-\int_{0}^{l} \mathcal{E}_{1}(x, t) d x .
$$

Substitution of Eq. 2 into Eq. 20 and specializing this to a "short-circuit" solution, where the electrical impedance between the target impact face and back face is negligible (see Fig. 1), results in

$$
V_{1}(t)=0=-h\left[u_{1}(l, t)-u_{1}(0, t)\right]+l D_{1}(t) / \epsilon
$$

where the quantity in brackets $\left[u_{1}(l, t)-u_{1}(0, t)\right]$ in Eq. 21 represents the relative motion of the target surfaces. Taking the Laplace transform of Eq. 21 and substitution of Eq. 17 results in

$$
\bar{V}_{1}(s)=0=\frac{h}{s}\left[\bar{F}_{1}(s)\left(1-e^{\frac{-s l}{c_{1}}}\right)+\bar{G}_{1}(s)\left(1-e^{\frac{s l}{c_{1}}}\right)\right]+l \bar{D}_{1}(s) / \epsilon .
$$

Three equations are required to find the three unknown functions $\bar{F}_{1}(s), \bar{G}_{1}(s)$, and $\bar{D}_{1}(s)$. The first is given by Eq. 21, the second is given by the boundary condition between the target and the half-space derived elsewhere, ${ }^{11}$

$$
\sigma_{1}(l, t)=z_{h} v_{1}(l, t)
$$

and the third equation is given by a newly derived ${ }^{11}$ impact boundary condition,

$$
z_{0} v_{1}(0, t)+\sigma_{1}(0, t)=z_{0} V_{0}
$$

Although not shown here, the elastodynamic impact boundary conditions (Eqs. 23 and 24) are also applicable to the piezoelectric impact problem for a homogeneous, elastic, and semi-infinite flyer and half-space backing the target. Substitution of Eqs. 17 through 19 into the Laplace transforms of Eqs. 21, 23, and 24 permits unique determination of functions $\bar{F}_{1}(s), \bar{G}_{1}(s)$, and 
$\bar{D}_{1}(s)$. Substitution of these functions into Eqs. 18, 19, and 22 allows determination of the stress, velocity, and displacement current by using numerical ILT algorithms. PZT-4 target mechanical and electrical properties ${ }^{13}$ are given in Table 1 . The elastic properties for the flyer and half-space are identical and similar to that of a "non-piezoelectric" steel material.

Table 1. Mechanical and electrical properties.

\begin{tabular}{lcc}
\hline Constant & PZT-4 & Steel \\
\hline$C^{E}(G P a)$ & 115.41 & 314.89 \\
\hline$z_{0}=\rho_{0} c_{0}\left(\mathrm{~kg} \mathrm{~m}^{-2} / \mathrm{s}^{2}\right)$ & - & $7850 \times 5110$ \\
\hline$z_{1}=\rho_{1} c_{1}\left(\mathrm{~kg} \mathrm{~m}^{-2} / \mathrm{s}^{2}\right)$ & $7500 \times 4533$ & - \\
\hline$z_{h}=z_{0}\left(\mathrm{~kg} \mathrm{~m} \mathrm{~m}^{-2} / \mathrm{s}^{2}\right)$ & - & $7850 \times 5110$ \\
\hline$\epsilon_{0}($ farads $/ \mathrm{m})$ & $8.854187817 \mathrm{e}-12$ & - \\
\hline$\epsilon_{r}$ & 663.2 & - \\
\hline$\epsilon=\epsilon_{0} \epsilon_{r}($ farads $/ \mathrm{m})$ & $5.87209736 \mathrm{e}-09$ & - \\
\hline$e\left(\mathrm{coul} / \mathrm{m}^{2}\right)$ & 15.08 & - \\
\hline$h=e / \epsilon(\mathrm{V} / \mathrm{m})$ & $2.5680773 \mathrm{e}+09$ & - \\
\hline$C^{D}=C^{E}+e h(\mathrm{GPa})$ & 154.136 & - \\
\hline
\end{tabular}

Solutions in the time domain are illustrated in Figs. 2, 3, and 4 and are found by numerical inversion of the Laplace transform using the DAC algorithm, ${ }^{18}$ modified by Laverty and Gazonas $^{12}$ to mitigate the effects of Gibbs' phenomenon using the so-called Lanczos $\sigma$-factors with 2048 terms in the Fourier series and a tolerance equal to $10^{-5}$. A general compiled Mathematica code for the numerical inversion of functions with jump discontinuities appears in the Appendix. The stress in the target in the Laplace domain $s$ is given by the function containing the exponentials in Eq. A-1. This same code works equally well for continuous functions and users need only replace the last expression in Eq. A-1 with their own Laplace-domain expression, as well as listing the constants appearing in their expression at the beginning of the Module command. Eq. A-2 computes the numerical ILT of the function Stress and stores these values in the function $\Sigma$. The Mathematica compile function executes this code in less than $8 \mathrm{~s}$ on a Dell laptop with a 64-bit OS and 8 GB of RAM running on an Intel(R) Core(TM) i5 CPU, M 560 at $2.67 \mathrm{GHz}$. The same code executes in $135 \mathrm{~s}$ using uncompiled Mathematica code. Eq. A-3 plots the normalized stress $\Sigma$ at the center of the piezoelectric target (see Fig. A-1); the stress is normalized by the initial elastic jump given by the expression sjump. Figs. 2, 3, and 4 also illustrate solutions to the piezoelectric impact problem using a FDTD method and COMSOL Multiphysics, a commercial FE code,${ }^{13}$ both of which exhibit wave dispersion effects. 


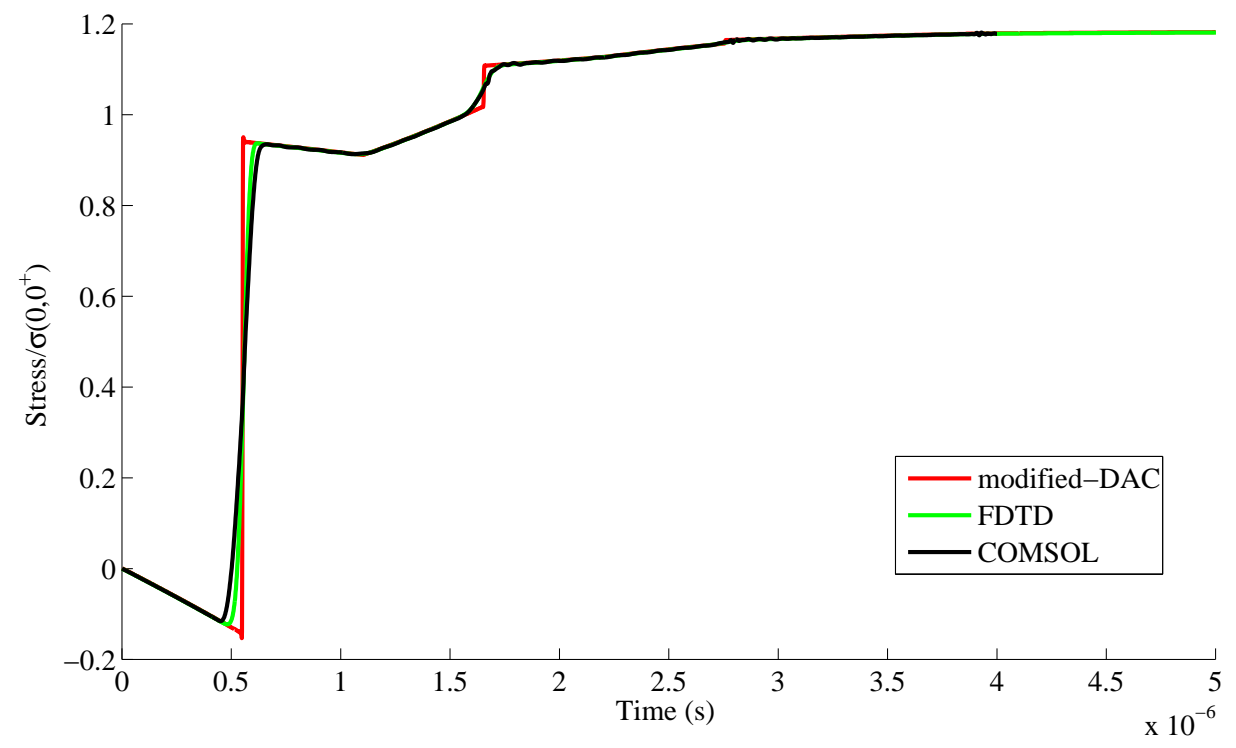

Fig. 2. Normalized stress history at the center of a 5- $m m$-thick piezoelectric target (PZT-4) backed by a semi-infinite non-piezoelectric elastic half-space under impact by a semi-infinite non-piezoelectric elastic flyer with initial velocity $V_{0}=5 \mathrm{~m} / \mathrm{s}$; the normalization factor is the initial elastic stress jump at impact given by $\sigma\left(0,0^{+}\right)=\frac{z_{0} z_{1 E}}{z_{0}+z_{1 E}} V_{0}$ where $z_{1 E}$ is the "elastic-only" impedance of the target, and

$z_{1 E}=\rho_{1} c_{1 E}=7500 \times 3922.8 c_{1 E}=\sqrt{C^{E} / \rho_{1}}$, see Table 1. 


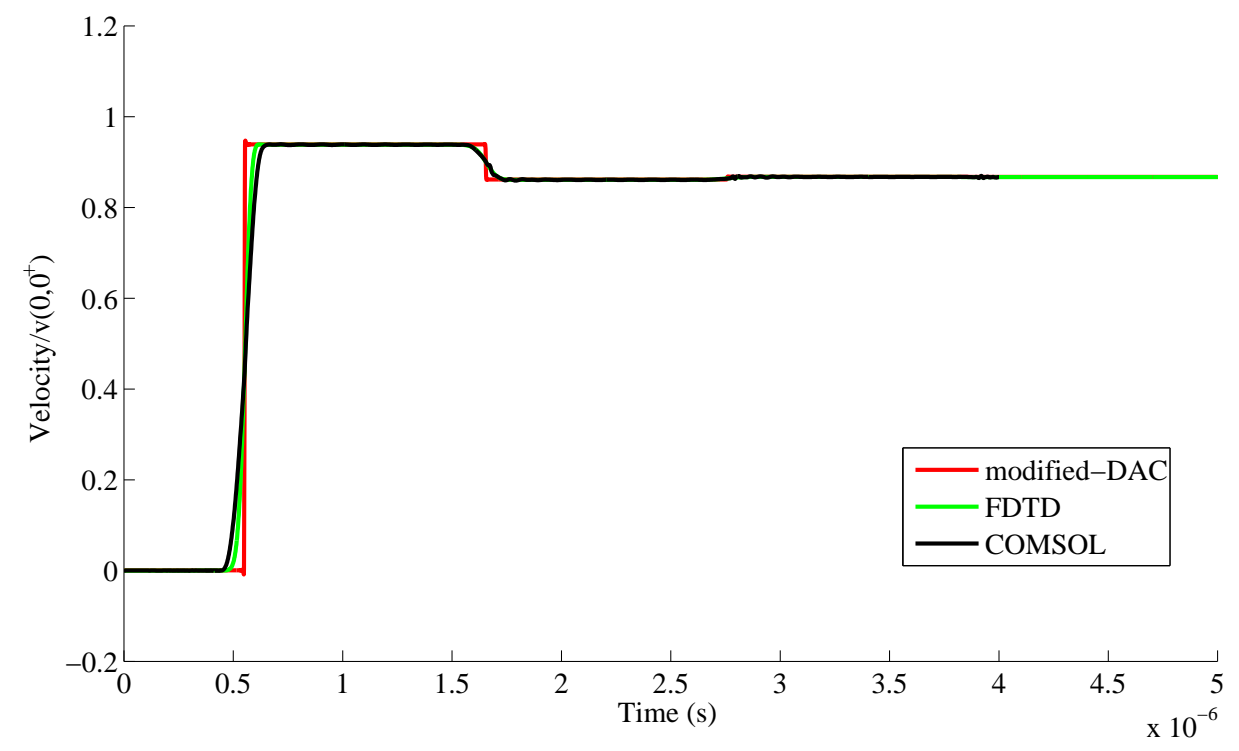

Fig. 3. Normalized velocity history at the center of a 5- $m m$-thick piezoelectric target (PZT-4) backed by a semi-infinite non-piezoelectric elastic half-space under impact by a semi-infinite non-piezoelectric elastic flyer with initial velocity $V_{0}=5 \mathrm{~m} / \mathrm{s}$; the normalization factor is the initial elastic velocity jump at impact given by $v\left(0,0^{+}\right)=\frac{z_{0}}{z_{0}+z_{1 E}} V_{0}$, where $z_{1 E}$ is the "elastic-only" impedance of the target, and

$z_{1 E}=\rho_{1} c_{1 E}=7500 \times 3922.8 c_{1 E}=\sqrt{C^{E} / \rho_{1}}$, see Table 1. 


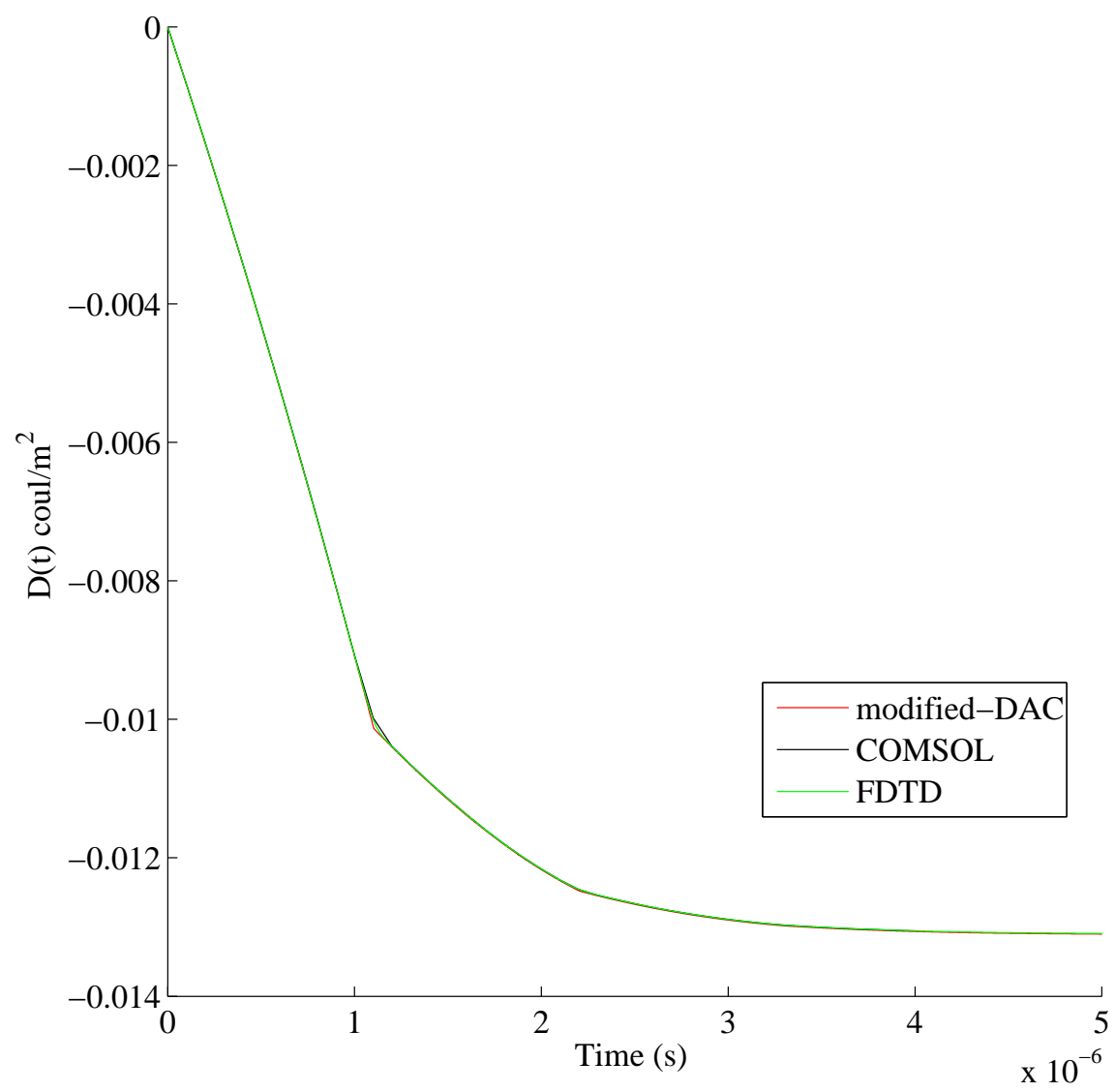

Fig. 4. Displacement current time history at the center of a 5-mm-thick piezoelectric target (PZT-4) backed by a semi-infinite non-piezoelectric elastic half-space under impact by a semi-infinite non-piezoelectric elastic flyer with initial velocity $V_{0}=5 \mathrm{~m} / \mathrm{s}$; the asymptotic displacement current $D_{1}(\infty)=-\frac{z_{0} z_{h} h \epsilon}{\left(z_{0}+z_{h}\right)\left(c_{1} z_{1}-h^{2} \epsilon\right)} V_{0}=-0.0131$. 


\section{Discussion and Conclusions}

The numerical solutions reveal the nearly instantaneous appearance of a tensile stress wave at the center of the target, which increases linearly in amplitude (see Fig. 2), and is formed by the $\mathcal{E}$-field induced polarization of the material in the initially unstressed region of the target. For nonlinear piezoelectric media, Chen et al. ${ }^{1}$ report that this wave cannot be a shock wave but must be an acceleration wave since the wave speed depends upon a time-dependent displacement current $D(t)$ (see Chen et al. ${ }^{1}$ equation (4.12)). The nearly instantaneous appearance of the stress wave occurs since the speed of light is about five orders of magnitude greater than the longitudinal wave speed in the target. The tensile stress wave is eventually overcome by the impact-induced compressive stress wave at about $0.5 \mu \mathrm{s}$. We are not aware of any other "true" elastodynamic impact solutions in the literature, which correctly treat the occurrence of multiply reflected transient waves in piezoelectric targets.

Numerical time-domain solutions of stress, velocity, and displacement current at the center of a 5-mm-thick PZT-4 target are obtained via a modified-DAC algorithm, and compare well with those obtained using both a FDTD method and the commercial FE code COMSOL Multiphysics. ${ }^{13}$ Although not shown in this report, the final value theorem (FVT) can be used to verify that the asymptotic (long-time) stress and velocity in the piezoelectric target are independent of the target's elastic or piezoelectric properties and only the elastic properties of the flyer and half-space, a result also proven using the time-domain solutions obtained for purely elastic targets. ${ }^{11}$ The FVT is also used to determine a closed-form expression for the asymptotic displacement current,

$$
D_{1}(\infty)=-\frac{z_{0} z_{h} h \epsilon}{\left(z_{0}+z_{h}\right)\left(c_{1} z_{1}-h^{2} \epsilon\right)} V_{0}=\frac{\sigma_{1}(x, \infty)}{\left(h-\frac{c_{1} z_{1}}{h \epsilon}\right)}
$$

illustrated in Fig. 4, where $\sigma_{1}(x, \infty)$ is the asymptotic target stress. ${ }^{11}$ 


\section{References}

1. Chen PJ, Davison L, McCarthy MF. Electrical responses of nonlinear piezoelectric materials to plane waves of uniaxial strain. J. Appl. Phys. 1976;47(11):4759-4764.

2. Graham RA. Second and third order piezoelectric stress constants of lithium niobate as determined by the impact loading technique. J. Appl. Phys. 1977;48(6):2153-2163.

3. Wang X, Dai HL. Dynamic focusing-effect of piezoelectric fibers subjected to radial impact. Compos. Struct. 2007;78(4):567-574.

4. Davison L. Fundamentals of Shock Wave Propagation in Solids. Heidelberg, Germany: Springer-Verlag; 2008.

5. Redwood M. Transient performance of a piezoelectric transducer. J. Acous. Soc. Am. 1961;33(4):527-536.

6. Steutzer OM. Multiple reflections in a free piezoelectric plate. J. Acous. Soc. Am. 1967;42(2):502-508.

7. Clayton JD. Modeling nonlinear electromechanical behavior of shocked silicon carbide. J. Appl. Phys. 2010;107.

8. Graham RA. Strain dependence of longitudinal piezoelectric, elastic, and dielectric constants of x-cut quartz. J. Appl. Phys. 1972;6(12):4779-4792.

9. Lysne PC. One dimensional theory of polarization by shock waves: Application to quartz gauges. J. Appl. Phys. 1972;43(2):425-431.

10. Scheidler MJ, Gazonas GA. Analytical and computational study of one-dimensional impact of graded elastic solids. In: Furnish M, editor. Shock Compression of Condensed Matter-2001; AIP Conference Proceedings 23; New York: American Institute of Physics; 2002. p. 689-692.

11. Gazonas GA, Scheidler MJ, Velo AP. Exact analytical solutions for elastodynamic impact. 2014;submitted.

12. Laverty R, Gazonas GA. An improvement to the Fourier series method for inversion of Laplace transforms applied to elastic and viscoelastic waves. Int. J. Comput. Meth. 2006;3(1):57-69. 
13. COMSOL Multiphysics Reference Manual, version 4.4. Burlington, MA: COMSOL, Inc.; 2013.

14. Standards on piezoelectric crystals. In: Proceedings of the I.R.E.; New York: The Institute of Radio Engineers; 1949. p. 1378-1395.

15. Cady WG. Piezoelectricity, volume one. New York: Dover Publications; 1964.

16. Lorrain P, Corson DR. Electromagnetic Fields and Waves. 2nd ed. New York: W.H. Freeman \& Co.; 1970.

17. Sternberg WJ, Smith TL. The Theory of Potential and Spherical Harmonics. Toronto, Canada: University of Toronto Press; 1964.

18. Crump KS. Numerical inversion of Laplace transforms using a Fourier series approximation. J. ACM 1976;23(1):89-96.

19. Mathematica Edition: version 9.0. Champaign, IL: Wolfram Research; 2013. 
INTENTIONALLY LEFT BLANK. 


\section{Appendix. Mathematica Code for Determining the Numerical ILT Using the Modified-DAC Algorithm}

The following is the Mathematica ${ }^{19}$ code for determining the numerical inverse Laplace transform (ILT) using the modified Dubner-Abate-Crump (DAC) algorithm. ${ }^{12}$ Figure A-1 shows the normalized stress time history of the target.

$$
\begin{aligned}
& \text { Stress }=\mathbf{C o m p i l e}\left[\left\{\left\{s, \_C o m p l e x\right\}\right\}, \operatorname{Module}[\{l, x, e o, C e, e r s, \epsilon 2, \epsilon, h, M, \rho, c 1, V 0 \text {, }\right. \\
& z 0, z 1, z h, a, b, c\}, l=5 * 10^{-3} ; x=l / 2 ; e o=8.854187817 * 10^{-12} ; C e=115.41 * 10^{9} \text {; } \\
& \text { ers }=663.2 ; \epsilon 2=15.08 ; \epsilon=e o * e r s ; h=\epsilon 2 / \epsilon ; M=C e+\epsilon 2 * h ; \rho=7500 ; \\
& c 1=\sqrt{\frac{M}{\rho}} ; V 0=5 ; z 0=7850 * 5110 ; z 1=\rho * c 1 ; z h=z 0 ; \\
& a=\frac{(z 0-z 1)(z 1-z h)}{(z 0+z 1)(z 1+z h)} ; b=z 0+2 z 1+z h ; c=\frac{z 0-2 z 1+z h}{b} ; \\
& \frac{V 0 z 0 e^{-\frac{s x}{c 1}}}{s}\left[\frac{\left(2 z 1 e^{\frac{s(l+x)}{c 1}}+z 1 e^{\frac{s(l+2 x)}{c 1}}+z 1 e^{\frac{l s}{c 1}}-(z 1-z h) e^{\frac{s x}{c 1}}\right)}{b\left(-e^{\frac{2 l s}{c 1}}+\frac{4 z 1 e^{\frac{l s}{c 1}}}{b}+c\right)+\frac{(z 0+z 1)(z 1+z h)}{h^{2} \epsilon} l s\left(e^{\frac{2 l s}{c 1}}+a\right)}\right. \\
& \left.\left.\left.+\frac{\left(-e^{\frac{s(2 l+x)}{c 1}}(z 1+z h)-z 1 e^{\frac{2 s x}{c 1}}\left(1+l s \frac{(z 1-z h)}{h^{2} \epsilon}\right)+z 1 e^{\frac{2 l s}{c 1}}\left(l s \frac{(z 1+z h)}{h^{2} \epsilon}-1\right)\right)}{b\left(-e^{\frac{2 l s}{c 1}}+\frac{4 z 1 e^{\frac{l s}{c 1}}}{b}+c\right)+\frac{(z 0+z 1)(z 1+z h)}{h^{2} \epsilon} l s\left(e^{\frac{2 l s}{c 1}}+a\right)}\right]\right]\right]
\end{aligned}
$$


$\Sigma=$ Compile $[\{\}$, Module $[\{$ sumcp, sumsp, $t f$, tol,$a$, tot $, c 0, \tau, d t, \sigma, \arg$, coe $f f s$, cospart, c1, c2, sinpart, kpd,t,kpt\};tf $=5 * 10^{-6} ;$ tol $=0.00001$;

$$
a=-\frac{\log [t o l]}{2 t f} ; \text { tot }=2048 ; c 0=\mathbf{S t r e s s}[a] ; \tau=1000 ; d t=\frac{t f}{\tau} ;
$$

Table $\left[\right.$ sumcp $=0 . ;$ sumsp $=0 . ; \mathbf{D o}\left[k p d=\frac{k \pi}{t o t} ; k p t=\frac{k \pi}{t f} ;\right.$

$$
\begin{aligned}
& \sigma=\frac{\operatorname{Sin}[k p d]}{k p d} ; \text { coeff } s=\operatorname{Stress}[a+i * k p t] ; c 1=\operatorname{Re}[\operatorname{coeff} s] ; \mathrm{c} 2=\operatorname{Im}[\text { coeff } s] ; \\
& \text { cospart }=\sigma c 1 \operatorname{Cos}[t * k p t] ; \text { sumcp }=\text { cospart }+ \text { sumcp; } \\
& \text { sinpart }=\sigma \mathrm{c} 2 \operatorname{Sin}[t * k p t]) ; \operatorname{sumsp}=\operatorname{sinpart}+\operatorname{sumsp},\{k, 1, \text { tot }\}] \text {; } \\
& \left.\left.\frac{\operatorname{Exp}[a * t]\left(\frac{c 0}{2}+s u m c p-s u m s p\right)}{t f},\{t, d t, t f, d t\}\right]\right],
\end{aligned}
$$

CompilationOptions $\rightarrow\{$ "InlineExternalDefinitions“" $\rightarrow$ True,

$$
\text { "InlineCompiledFunctions“ } \rightarrow \text { True }\}]
$$

$$
\begin{gathered}
V 0=5 ; \rho=7500 ; z 0=\rho * 5110 ; C e=115.41 * 10^{9} ; z n=\sqrt{\rho * C e} ; \\
\text { sjump }=V 0 \frac{z 0 z n}{z 0+z n} ; \text { ListPlot }[\Sigma[] / \text { sjump, Joined } \rightarrow \text { True }, \\
\text { BaseStyle } \rightarrow\{\text { FontFamily } \rightarrow \text { "Times“, FontSize } \rightarrow 12\}, \\
\text { PlotRange } \rightarrow \text { Full, DataRange } \rightarrow\left\{0,5 * 10^{-6}\right\}, \\
\text { AxesLabel } \left.\rightarrow\left\{\text { "Time (s)", " } \frac{\sigma(l / 2, t)}{\sigma(0,0)} \text { “ }\right\}\right]
\end{gathered}
$$




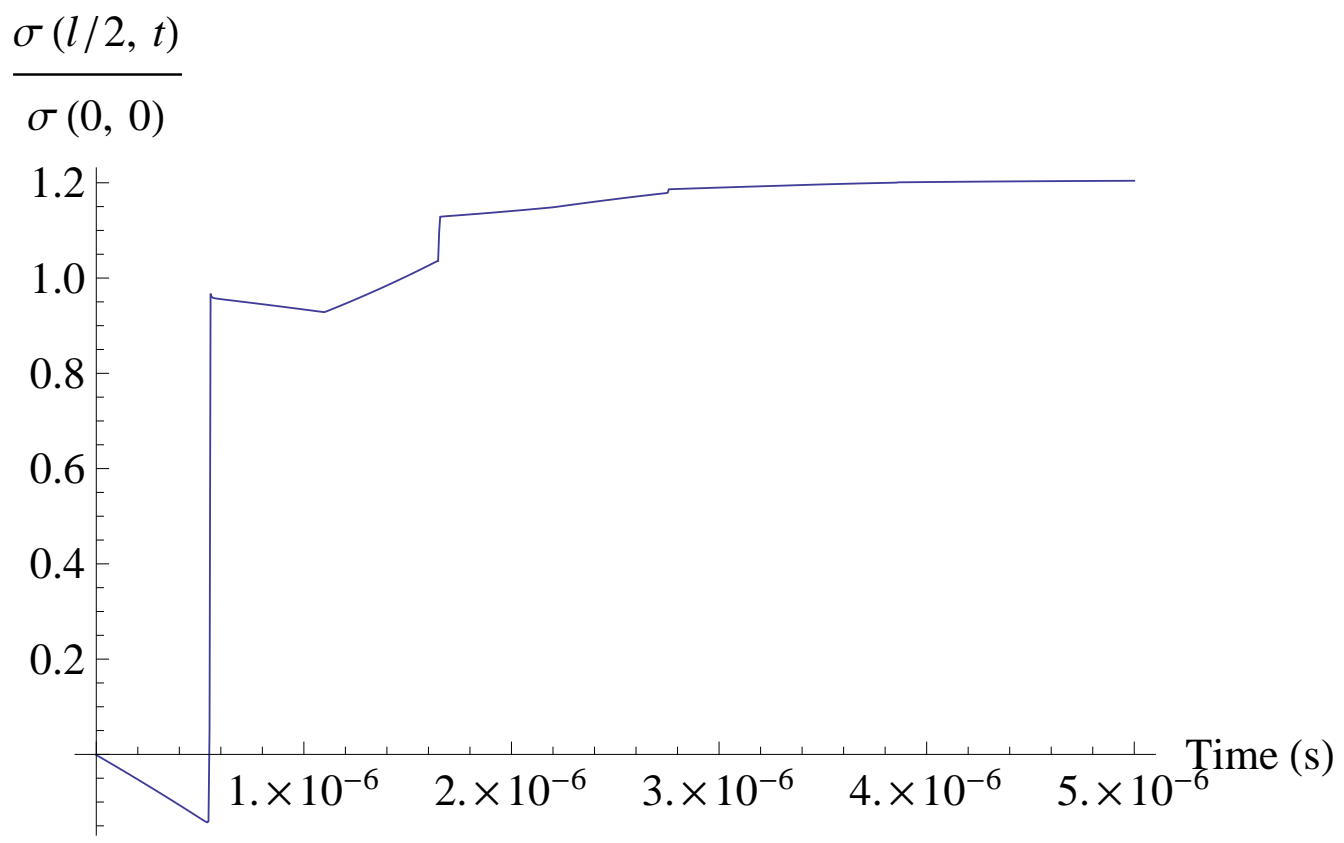

Fig. A-1. Normalized stress time history at the center of a 5-mm-thick piezoelectric target (PZT-4) backed by a semi-infinite non-piezoelectric elastic half-space under impact by a semi-infinite non-piezoelectric elastic flyer with initial velocity $V_{0}=5 \mathrm{~m} / \mathrm{s}$. 


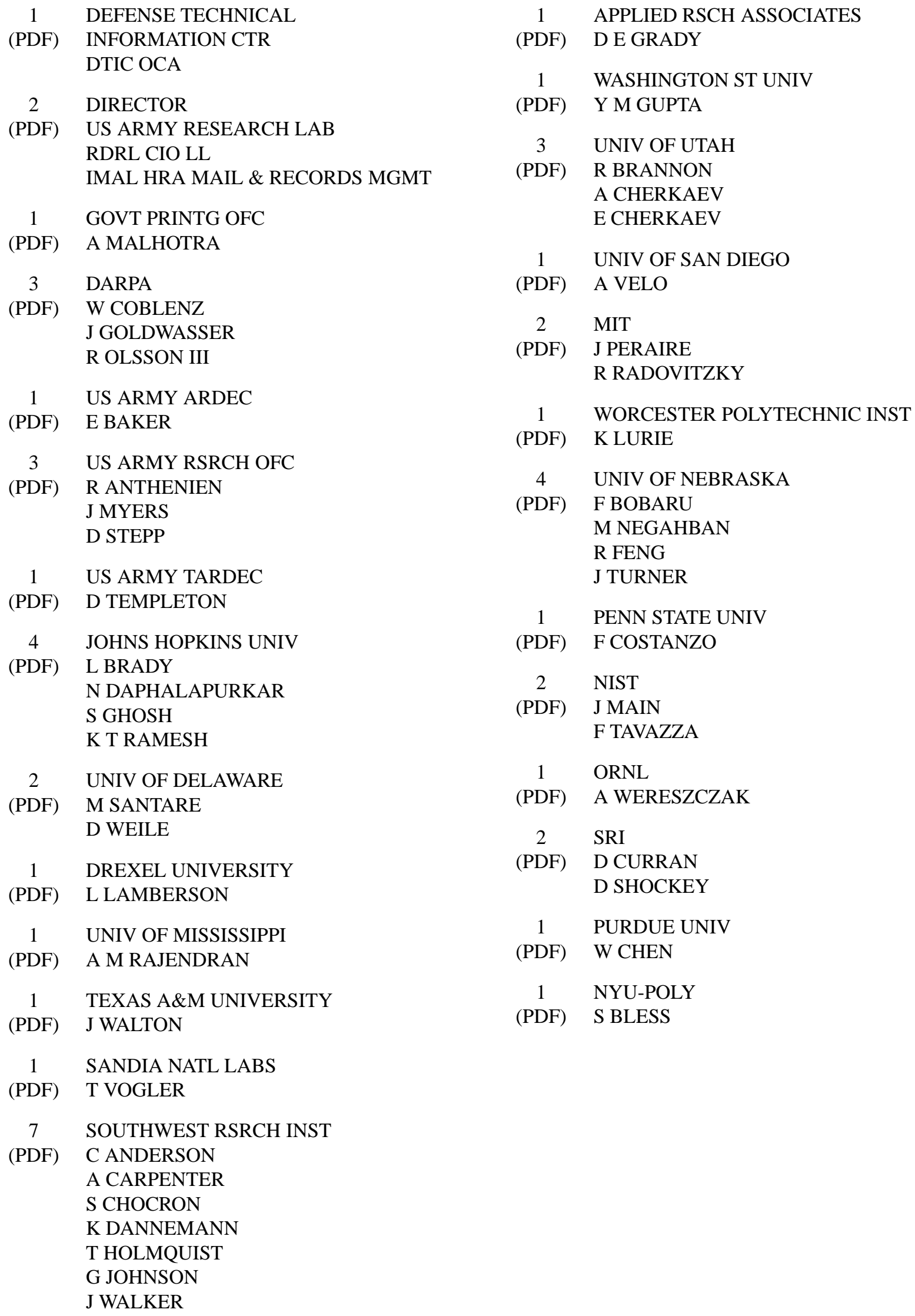




\begin{tabular}{|c|c|}
\hline 95 & DIR USARL \\
\hline (PDF) & RDRL D \\
\hline & O OCHOA \\
\hline & RDRL CIH C \\
\hline & J CAZAMIAS \\
\hline & D GROVE \\
\hline & J KNAP \\
\hline & RDRL WM \\
\hline & P BAKER \\
\hline & B FORCH \\
\hline & S KARNA \\
\hline & J MCCAULEY \\
\hline & M VANLANDINGHAM \\
\hline & RDRL WML \\
\hline & M ZOLTOSKI \\
\hline & RDRL WML B \\
\hline & I BATYREV \\
\hline & J BRENNAN \\
\hline & E BYRD \\
\hline & S IZVEKOV \\
\hline & B RICE \\
\hline & R PESCE RODRIGUEZ \\
\hline & D TAYLOR \\
\hline & N TRIVEDI \\
\hline & N WEINGARTEN \\
\hline & RDRL WML D \\
\hline & P CONROY \\
\hline & M NUSCA \\
\hline & RDRL WML E \\
\hline & P WEINACHT \\
\hline & RDRL WML G \\
\hline & M BERMAN \\
\hline & W DRYSDALE \\
\hline & M MINNICINO \\
\hline & RDRL WML H \\
\hline & C MEYER \\
\hline & J NEWILL \\
\hline & D SCHEFFLER \\
\hline & B SCHUSTER \\
\hline & RDRL WMM \\
\hline & J BEATTY \\
\hline & R DOWDING \\
\hline & J ZABINSKI \\
\hline & RDRL WMM A \\
\hline & J SANDS \\
\hline & $\mathrm{J}$ TZENG \\
\hline & RDRL WMM B \\
\hline & P BARNES \\
\hline & T BOGETTI \\
\hline & R DOOLEY \\
\hline & C FOUNTZOULAS \\
\hline & G GAZONAS \\
\hline & D HOPKINS \\
\hline & B LOVE \\
\hline
\end{tabular}

\author{
P MOY \\ B POWERS \\ T WALTER \\ R WILDMAN \\ C YEN \\ J YU \\ RDRL WMM C \\ J LA SCALA \\ RDRL WMM D \\ R CARTER \\ B CHEESEMAN \\ RDRL WMM E \\ M BRATCHER \\ M COLE \\ J LASALVIA \\ P PATEL \\ J SINGH \\ RDRL WMM F \\ L KECSKES \\ H MAUPIN \\ T SANO \\ M TSCHOPP \\ RDRL WML G \\ J ANDZELM \\ J LENHART \\ A RAWLETT \\ RDRL WMP \\ D LYON \\ S SCHOENFELD \\ RDRL WMP A \\ J FLENIKEN \\ A PORWITZKY \\ RDRL WMP B \\ C HOPPEL \\ S SATAPATHY \\ M SCHEIDLER \\ A SOKOLOW \\ T WEERASOORIYA \\ RDRL WMP C \\ R BECKER \\ S BILYK \\ T BJERKE \\ D CASEM \\ J CLAYTON \\ D DANDEKAR \\ M GREENFIELD \\ B LEAVY \\ M RAFTENBERG \\ S SEGLETES \\ RDRL WMP D \\ R DONEY \\ D KLEPONIS \\ H MEYER \\ C RANDOW
}


J RUNYEON

S SCHRAML

B SCOTT

M ZELLNER

RDRL WMP E

$S$ BARTUS

RDRL WMP F

A FRYDMAN

N GNIAZDOWSKI

RDRL WMP G

N ELDREDGE

D KOOKER

S KUKUCK 

